\title{
The competition between fracture nucleation, propagation, and coalescence in dry and water-saturated crystalline rock
}

\author{
Jessica A. McBeck ${ }^{1}$, Wenlu Zhu ${ }^{2}$, and François Renard ${ }^{1,3}$ \\ ${ }^{1}$ Njord Centre, Department of Geosciences, University of Oslo, Oslo, Norway \\ ${ }^{2}$ Department of Geology, University of Maryland, College Park, USA \\ ${ }^{3}$ University Grenoble Alpes, University Savoie Mont Blanc, CNRS, IRD, IFSTTAR, ISTerre, Grenoble, France
}

Correspondence: Jessica McBeck (j.a.mcbeck@geo.uio.no)

Received: 30 June 2020 - Discussion started: 14 July 2020

Revised: 13 January 2021 - Accepted: 14 January 2021 - Published: 16 February 2021

\begin{abstract}
The continuum of behavior that emerges during fracture network development in crystalline rock may be categorized into three end-member modes: fracture nucleation, isolated fracture propagation, and fracture coalescence. These different modes of fracture growth produce fracture networks with distinctive geometric attributes, such as clustering and connectivity, that exert important controls on permeability and the extent of fluid-rock interactions. To track how these modes of fracture development vary in dominance throughout loading toward failure and thus how the geometric attributes of fracture networks may vary under these conditions, we perform in situ X-ray tomography triaxial compression experiments on low-porosity crystalline rock (monzonite) under upper-crustal stress conditions. To examine the influence of pore fluid on the varying dominance of the three modes of growth, we perform two experiments under nominally dry conditions and one under watersaturated conditions with $5 \mathrm{MPa}$ of pore fluid pressure. We impose a confining pressure of 20-35 MPa and then increase the differential stress in steps until the rock fails macroscopically. After each stress step of 1-5 MPa we acquire a three-dimensional (3D) X-ray adsorption coefficient field from which we extract the 3D fracture network. We develop a novel method of tracking individual fractures between subsequent tomographic scans that identifies whether fractures grow from the coalescence and linkage of several fractures or from the propagation of a single fracture. Throughout loading in all of the experiments, the volume of preexisting fractures is larger than that of nucleating fractures, indicating that the growth of preexisting fractures dominates the nucleation of new fractures. Throughout loading until close to macro-
\end{abstract}

scopic failure in all of the experiments, the volume of coalescing fractures is smaller than the volume of propagating fractures, indicating that fracture propagation dominates coalescence. Immediately preceding failure, however, the volume of coalescing fractures is at least double the volume of propagating fractures in the experiments performed at nominally dry conditions. In the water-saturated sample, in contrast, although the volume of coalescing fractures increases during the stage preceding failure, the volume of propagating fractures remains dominant. The influence of stress corrosion cracking associated with hydration reactions at fracture tips and/or dilatant hardening may explain the observed difference in fracture development under dry and water-saturated conditions.

\section{Introduction}

Fracture and fault networks develop through the nucleation of new fractures, the propagation of new and preexisting fractures, and the coalescence of neighboring fractures (e.g., Tapponnier and Brace, 1976; Nemat-Nasser and Horii, 1982; Atkinson, 1984; Olson, 1993; Lockner et al., 1991; Reches and Lockner, 1994; Martin and Chandler, 1994; Kawakata et al., 1997; Mansfield and Cartwright, 2001; Crider and Peacock, 2004; Jackson and Rotevatn, 2013). Formulations of linear elastic fracture mechanics (LEFM) can describe the potential for propagation of one or a few fractures within linear elastic material (e.g., Griffith, 1921; Irwin, 1957). However, such analytical formulations do not describe the coalescence behavior of fracture networks as they transi- 
tion from distributed, disperse networks comprised of many isolated, small fractures to more localized networks comprised of well-connected, larger fractures. Laboratory experiments provide rich examples of fracture networks coalescing from isolated, smaller fractures to larger, connected fractures (e.g., Cartwright-Taylor et al., 2020). As some fractures grow longer, their rate of propagation may increase with increasing fracture length and thus stress intensity factor (Jaeger et al., 1979). These faster-growing fractures may then cause the rate of growth of smaller fractures to slow or stop. These varying rates of fracture growth produce the transition from distributed to localized fracture networks. This transition includes a continuum of fracture development that may be divided into three end-member modes of fracture growth: (1) nucleation, (2) isolated propagation, and (3) coalescence.

The aim of this work is to provide experimental constraints on the stress and fluid conditions that promote the dominance of one mode of fracture network development over another. Identifying which of these modes dominates the others under varying conditions may be critical for accurate assessment of fracture network development. For example, if nucleation is the dominant mode of fracture development rather than isolated propagation, then using metrics that identify sites of potential fracture nucleation may be more accurate than using metrics that predict the conditions under which a preexisting fracture will grow. Metrics that indicate regions in which fractures may nucleate include the strain energy density, maximum Coulomb stress, maximum magnitude of shear stress, and highest tensile stress or least compressive stress (e.g., Jaeger et al., 1979; Atkinson, 1987; Du and Aydin, 1993). Previous analyses have used some of these metrics to predict the direction of fracture growth from a preexisting fracture tip (e.g., Olson and Cooke, 2005; Okubo and Schulz, 2005; Fattaruso et al., 2016). However, these metrics can lead to conflicting predictions about both the sites of new fracture nucleation and the direction of fracture growth (e.g., Madden et al., 2017; McBeck et al., 2017, 2020b). If preexisting fracture propagation is the dominant mode of development rather than fracture nucleation, then metrics that determine the conditions under which preexisting fractures will grow, such as the critical stress intensity factor (Isida, 1971), and the direction of fault growth, such as Coulomb shear stress, tensile stress, and energy optimization (e.g., Pollard and Aydin, 1988; Müller, 1998; Mary et al., 2013; Madden et al., 2017; McBeck et al., 2017), may provide more accurate predictions of fault network development than nucleation criteria. Thus, determining which mode dominates deformation under varying confinement and fluid conditions may help identify analyses suitable for successful prediction of fracture network development.

The mode of fracture growth that dominates deformation may also influence the permeability of the network and effectivity of fluid-rock interactions because these modes can control the connectivity, tortuosity, and total fracture surface area of the network (e.g., Hickman et al., 1995). In particu- lar, if a fracture network is dominated by many isolated fractures that propagate independently, it may host lower connectivity, greater tortuosity, and higher fracture surface area available for chemical reactions than a network dominated by several connected fractures that form via coalescence. The connectivity, tortuosity, and available fracture surface area may influence the effective permeability and the rate and extent of fluid-rock interactions (Hickman et al., 1995; Blanpied et al., 1998; Lamy-Chappuis et al., 2014; Frery et al., 2015). In particular, fluid-rock interactions in a rock with many distributed small fractures that hosts greater fracture surface area may be more effective than in one with a few large fractures with lower surface area, depending on the permeability of the rock and whether the reaction is diffusion-controlled (e.g., Renard et al., 2000). A distributed fracture network comprised of many unconnected fractures may produce lower permeability than a more localized and connected fracture network. This difference in permeability may then influence the ability of fluid to access rock surfaces and react with them. In turn, reactions that dissolve the host rock or precipitate new material can influence the porosity and permeability (e.g., Sausse et al., 2001; Tenthorey et al., 2003; Lamy-Chappuis et al., 2014; Xing et al., 2018). Thus, identifying the conditions under which coalescence or isolated propagation dominates may help assess the efficiency of geothermal energy and unconventional fossil fuel productions and identify sites ideal for waste disposal or $\mathrm{CO}_{2}$ sequestration (e.g., Saeedi et al., 2016; Cui et al., 2018).

To investigate the relative contributions of the three endmember deformation modes to fracture network development, we quantify the evolution of 3D fracture networks in monzonite rock samples undergoing brittle failure using in situ dynamic X-ray synchrotron microtomography. We conducted three triaxial deformation experiments at room temperature and confining pressures of 20-35 MPa. In two of the experiments, the sample was deformed at nominally dry conditions. In the third experiment, the sample was saturated with deionized water and deformed at a constant pore fluid pressure of $5 \mathrm{MPa}$ under drained conditions. In the experiments, we increased the maximum principal (compressive) stress $\sigma_{1}$ in distinct steps of 1-5 MPa until macroscopic failure while we applied constant intermediate and minimum principal stresses of $\sigma_{2}=\sigma_{3}=20-35 \mathrm{MPa}$ (Fig. 1). After each differential stress $\left(\sigma_{1}-\sigma_{2}\right)$ increase, we acquired a microtomographic scan of the deforming rock at in situ stress conditions. From these scans, we obtained the evolving three-dimensional (3D) fracture networks within the samples (Fig. 2). We developed a novel method of tracking the growth of fractures that enables distinguishing between fractures that grow via isolated propagation and those that grow from the coalescence of several fractures. These new methods enable quantitative comparison of the competing influences of (1) nucleation and preexisting propagation, (2) isolated propagation and coalescence, and (3) local stress perturbations. Our analyses show that these competitions evolve 

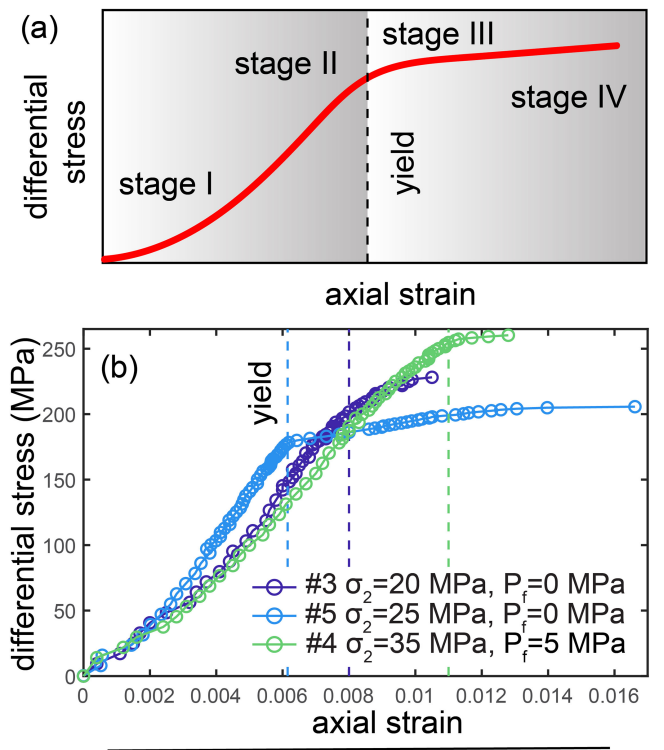

(c)

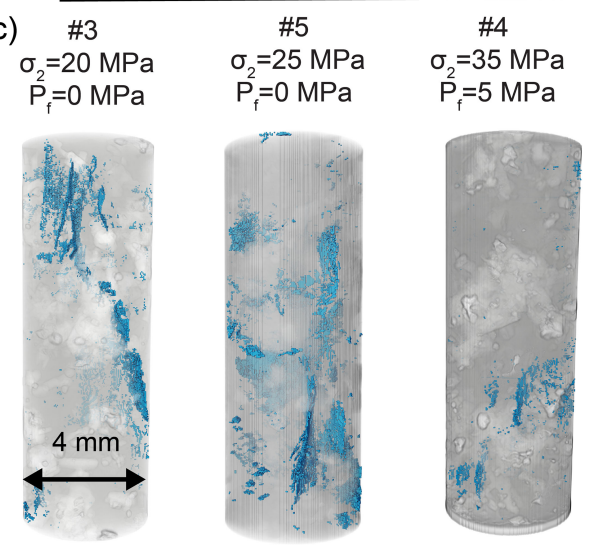

Figure 1. Macroscopic behavior of each experiment produced by fracture network development. (a) Macroscopic stages of deformation. Stage I is the initial nonlinear stage corresponding to the closure of preexisting defects. Stage II includes the quasi-linear relationship between stress and strain. Stage III occurs when deformation behavior deviates significantly from linearity. The yield point marks the boundary between stages II and III. Stage IV occurs close to macroscopic failure, when the effective elastic modulus is near zero. The timing of the transition between stages I-II and stages IIIIV remains approximate in this analysis. (b) Differential stress and axial strain relationships of the three experiments: no. 3 , no. 4 , and no. 5. Circles show the conditions when a tomogram was acquired. The applied confining stress and pore fluid pressure increase from monzonite in no. $3\left(\sigma_{2}=20 \mathrm{MPa}, P_{\mathrm{f}}=0\right)$, no. $5\left(\sigma_{2}=25 \mathrm{MPa}\right.$, $\left.P_{\mathrm{f}}=0\right)$, and no. $4\left(\sigma_{2}=35 \mathrm{MPa}, P_{\mathrm{f}}=5 \mathrm{MPa}\right)$. (c) Fracture geometry in the final scan in all three experiments. Fractures are shown in blue, and minerals are shown with transparent grey and white. The fracture network geometry in the last scan acquired before macroscopic failure includes longer, more volumetric, and more connected fractures in the experiments with $\sigma_{2}=20-25 \mathrm{MPa}$ and $P_{\mathrm{f}}=0$ (no. 3, no. 5) than in the experiment with $\sigma_{2}=35 \mathrm{MPa}$ and $P_{\mathrm{f}}=5 \mathrm{MPa}$ (no. 4).

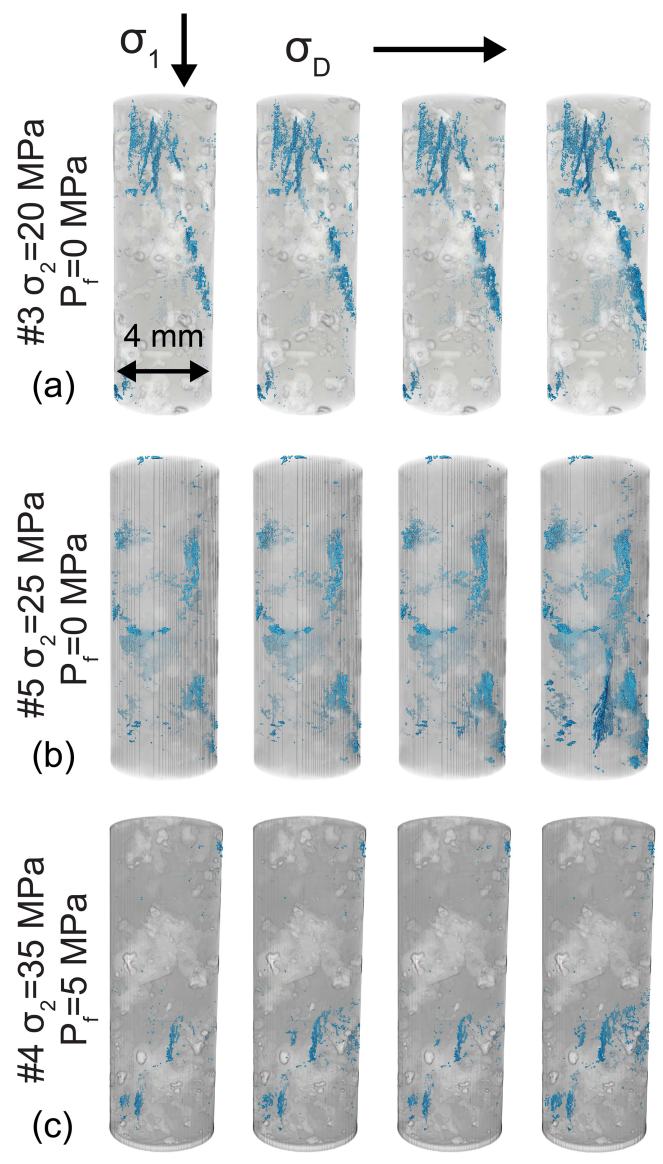

Figure 2. Evolving fracture networks in the final four loading steps of each experiment before system size failure.

toward macroscopic failure and depend on the stress states and interstitial fluid.

\section{Methods}

\subsection{In situ X-ray tomography}

We performed three triaxial deformation experiments with in situ dynamic X-ray synchrotron microtomography at beamline ID19 at the European Synchrotron and Radiation Facility (ESRF). We deformed monzonite cylinders $1 \mathrm{~cm}$ in height and $0.4 \mathrm{~cm}$ in diameter using the HADES apparatus (Renard et al., 2016). Monzonite is an igneous crystalline rock with similar mechanical properties to granite. Using the porosity measured in the tomograms, the initial porosity of each rock core is close to zero. This monzonite has a mean grain size of $450 \mu \mathrm{m}$ (Aben et al., 2016). The large grain size relative to the sample size may cause the representative elementary volume (REV) of the system to approach the size of the sample. The question of the appropriate REV size is critical to address in order to aid reproducibility of the results, but it is difficult to estimate. The following analysis, and previous work 
describing these experiments (Renard et al., 2018, 2019b), finds general similarities in fracture network development in these three experiments, suggesting the reproducibility and robustness of the results.

In each experiment, we imposed a constant confining pressure $\left(\sigma_{2}=\sigma_{3}\right)$ and then increased the axial stress $\left(\sigma_{1}\right)$ in steps until the rock failed macroscopically (Fig. 1). After each differential stress increment, we acquired a scan of the sample at in situ stress conditions with $6.5 \mu \mathrm{m}$ voxel resolution (Renard et al., 2016). We measured the axial shortening using a linear variable differential transformer displacement sensor. The duration of each scan is within $2 \mathrm{~min}$. Thus, the time required to increase the differential stress $(1 \mathrm{~min})$ and to acquire the scan $(2 \mathrm{~min})$, as well as the applied increase in axial stress per loading step (1-5 MPa), determines the loading rate of $1 \mathrm{MPa}$ every $3 \mathrm{~min}$ to $5 \mathrm{MPa}$ every $3 \mathrm{~min}$. The experiments were conducted at room temperature with three different confining pressures: $20 \mathrm{MPa}$ (experiment no. 3), $25 \mathrm{MPa}$ (no. 5), and $35 \mathrm{MPa}$ (no. 4). Macroscopic failure occurred in a sudden stress drop. The final scan was taken at a differential stress very close to the failure stress, typically $<0.5 \mathrm{MPa}$ below the failure stress. Experiment no. 3 and no. 5 were conducted at nominally dry conditions, while the sample was fully saturated in experiment no. 4. This sample was submerged in deionized water for $24 \mathrm{~h}$ under vacuum before the experiment to help ensure that the pore space was saturated. In experiment no. 4, a constant pore fluid pressure of $5 \mathrm{MPa}$ was maintained using two pore pressure pumps connected at each end of the sample (top and bottom). Experiment no. 4 is also unique in that we reached the axial stress limit of the device (200 MPa) preceding macroscopic failure, and thus we reduced the confining pressure in steps of $1 \mathrm{MPa}$ from 35 to $31 \mathrm{MPa}$ until the core failed. Consequently, the sample experienced $35 \mathrm{MPa}$ of confining pressure for 60 scans and stress steps, and then it experienced $34,33,32$, and $31 \mathrm{MPa}$ of confining pressure in the final four scans preceding failure. Renard et al. $(2018,2019 b)$ describe the experimental conditions in further detail. Renard et al. (2018) describe experiment no. 3 and no. 4. Renard et al. (2019b) analyze experiment no. 5. In the present study, we develop a new technique to follow the dynamics of fracture growth by categorizing this growth into three end-member modes of growth. The Xray tomography data from the three experiments are publicly available (Renard, 2017, 2018).

\subsection{Extraction of the fracture networks}

From the time series of 3D adsorption coefficient fields acquired throughout loading, we identify fractures and pores using a standard thresholding technique. The histogram of greyscale values from a tomogram of a porous rock tends to have two maxima indicative of the modes of the solid and air (or deionized water) populations (e.g., Renard et al., 2019a). The local minimum of this histogram then determines the threshold that indicates whether voxels are identified as pore

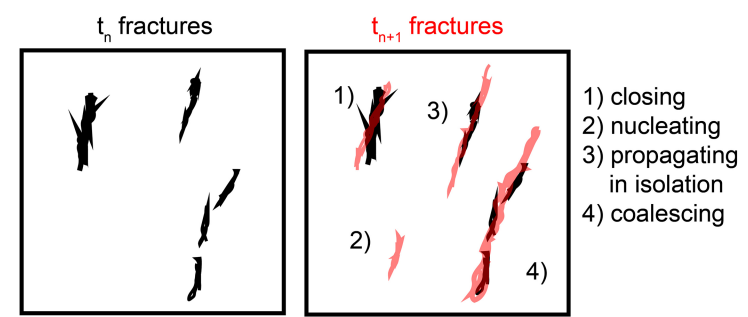

Figure 3. Modes of fracture network development captured by the algorithm. By tracking individual fractures in sequential scans, we can identify fractures that (1) close, (2) nucleate, (3) propagate in isolation, and (4) coalesce from one time to the next, $t_{n}$ to $t_{n+1}$.

space or solid. Segmenting the tomograms with this procedure yields 3D binary fields of zeros and ones that indicate whether a voxel is within or outside a fracture or pore. Because we employ the same threshold throughout loading in each experiment, the choice of the threshold has a similar effect for the entire time series of scans.

From the binary field, we extract individual fracture or pore objects by identifying groups of voxels that have 26-fold connectivity, the highest degree of connectivity in 3D. For each group of voxels, we calculate the covariance matrix and corresponding eigenvectors and eigenvalues, which describe the shape of each fracture using three principal orthogonal length scales corresponding to the eigenvectors. If the pore had an ellipsoidal shape, the three eigenvalues would represent the lengths of the three axes of the ellipsoid. We then use these eigenvalues to characterize the dimensions of fractures and pores in subsequent analyses. For all of the calculations using the fracture volume, we use the actual volume of the group of connected voxels. For all calculations that depend on the placement of the fractures in space, we use the three eigenvalues calculated from the covariance matrix. Because mineral grain boundaries do not exert a significant impact on the geometry of fractures in these monzonite cores, the three eigenvectors of the covariance matrix provide a close approximation of the true fracture.

\subsection{Identifying nucleating, propagating, and coalescing fractures}

After identifying the individual fractures at each loading step of an experiment, we track the fractures across several loading steps. In addition, we develop a method that links one or more fractures at the previous loading step $\left(t_{n}\right)$ to the next loading step $\left(t_{n+1}\right)$ (Fig. 3). This development is the central difference between this new method and the previous method of tracking fractures in X-ray tomography data developed by Kandula et al. (2019) and used in McBeck et al. (2019a). The previous method did not allow linking more than one fracture in $t_{n}$ to a fracture in $t_{n+1}$. Thus, Kandula et al. (2019) could identify when an individual fracture gained or lost volume from one loading step (and tomogram) to the next. However, 

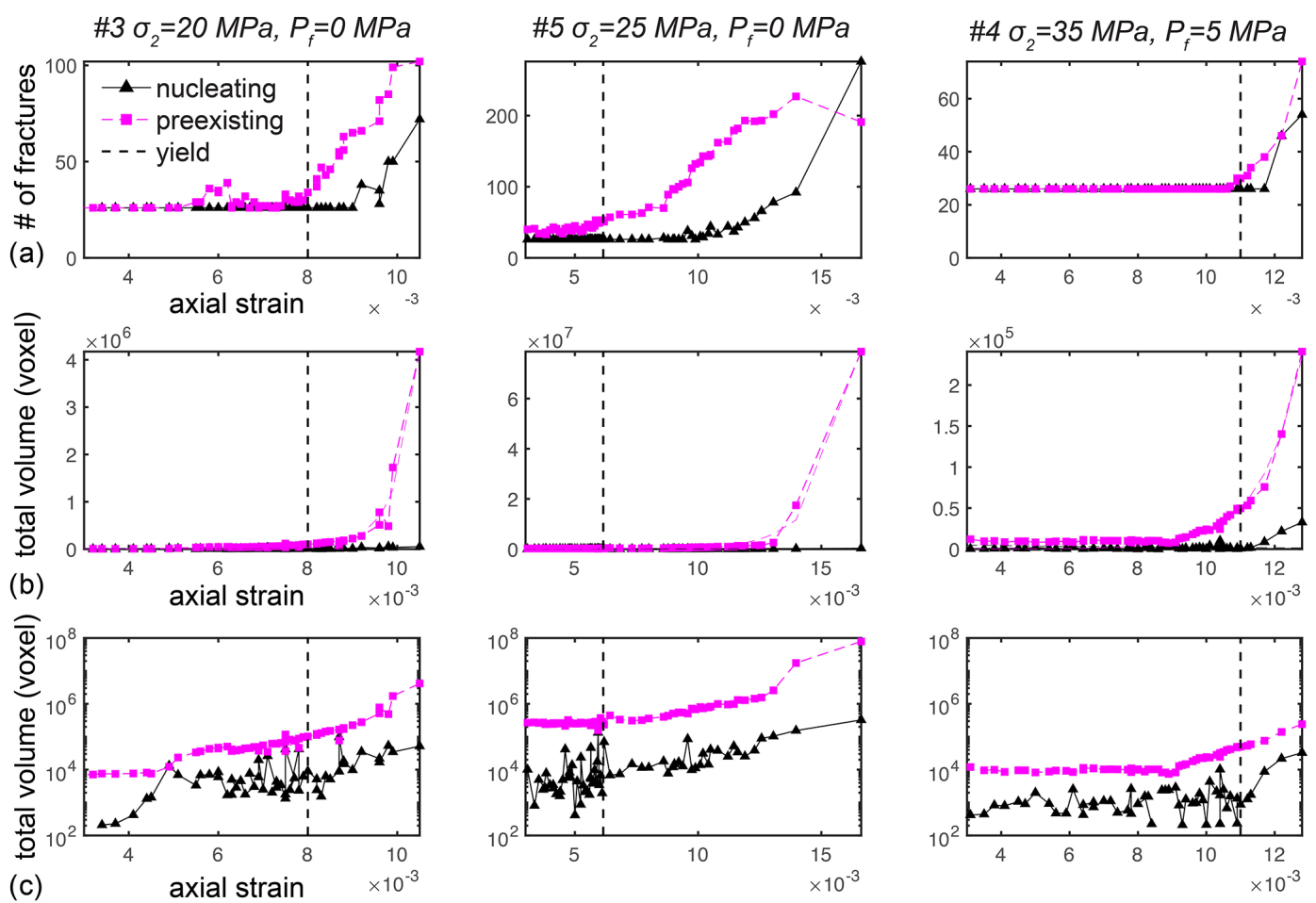

Figure 4. The competing influence of fracture nucleation and preexisting growth in each experiment. The applied effective pressure $\left(\sigma_{2}-P_{\mathrm{f}}\right)$ increases from left to right. (a) The number of fractures identified as nucleating or preexisting in each loading step. The total volume of the nucleating and preexisting fractures in linear (b) and log-linear (c) space. Dashed vertical lines show the axial strain at the macroscopic yielding point identified from the shallowing of the stress-strain curves (Figs. 1, S2), separating stages I-II and III-IV. The pink lines without markers (b) show the best-fit exponential functions of the data. The total volume of preexisting fractures exceeds the volume of newly nucleating fractures in the final loading steps preceding macroscopic failure, indicating the dominance of preexisting development rather than nucleation. The increase in the volume of nucleating fractures after yield is more significant in the water-saturated sample compared to the nominally dry samples.

this analysis could not differentiate between fractures that gained volume because one fracture propagated and opened or because several fractures propagated and linked to each other (i.e., coalesced).

We developed this new method of tracking fractures in order to examine the competing influence of fracture coalescence and isolated propagation (Fig. 3). Our method identifies one or more fractures in $t_{n}$ and one fracture in $t_{n+1}$ by searching for fractures in $t_{n}$ that are within five voxels of a fracture in $t_{n+1}$. We use the ellipsoidal approximations of the fractures to do this search. The limit of five voxels helps ensure that the algorithm identifies fractures that have shifted in space due to deformation. The appropriate value of this limit may differ in rocks that experience differing axial and radial strains in each loading step than those observed here. We only perform the analysis for fractures with volumes $>100$ voxels. This volume threshold helps exclude noise from the analysis. The appropriate volume threshold is likely different for rocks that host differing ranges of fracture volumes than those observed here. Varying the volume threshold from 100 to 500 voxels does not change the main trends described in the results (Fig. S1).
Determining whether a fracture is nucleating, propagating, or coalescing at a given time step depends on the spatial resolution of the tomogram and the amount of opening that the fracture accommodates. We may only detect fractures with apertures greater than the scan voxel size $(6.5 \mu \mathrm{m})$. Thus, the nucleating classification refers to newly identified fractures with apertures $>6.5 \mu \mathrm{m}$, which may have formed in a previous loading step with apertures $<6.5 \mu \mathrm{m}$.

\section{Results}

\subsection{Macroscopic mechanical behavior}

The global mechanical behavior captured in the differential stress and axial strain relationships indicates that the monzonite samples undergo the deformation stages typical for brittle materials under triaxial compression (e.g., Paterson and Wong, 2005). We may separate the macroscopic deformation behavior into four different stages (Fig. 1). Stage I is the initial nonlinear stage corresponding to closure of preexisting defects. Stage II includes a quasi-linear relationship 
between stress and strain. Stage III occurs when deformation behavior deviates significantly from linearity. The yield point marks the boundary between stages II and III. Stage IV occurs shortly before macroscopic failure, when the effective elastic modulus is near zero (Fig. 1). Figure 1 shows the axial strains when the initial shallowing occurs, which we refer to as the yield point in the subsequent text. We identify the yield point using the largest axial strain at which the difference between the observed differential stress and the differential stress predicted from a linear fit is less than $1 \%$ of the observed differential stress (Fig. S2). We note that we leave the timing of the transitions from stage I to II and from stage III and IV as only qualitative in the subsequent analysis, while the transition from stage II to III is more precisely defined as the yield point. The macroscopic failure of the rocks occurred in a sudden stress drop that either completely crushed the core (experiment no. 3 and no. 5) or allowed partial recovery of the core (no. 4). The macroscopic failure of experiment no. 4 included the formation of a system-spanning fracture network oriented approximately $30^{\circ}$ from $\sigma_{1}$ (Fig. 4 in Renard et al., 2018).

\subsection{Fracture nucleation and preexisting fracture propagation}

Here we assess the dominance of fracture nucleation relative to the growth of preexisting fractures throughout loading in the three experiments (Fig. 4). We track the number and total volume of fractures identified in a loading step that did (i.e., preexisting) and did not (i.e., nucleating) grow from a preexisting fracture identified in the previous loading step. In this and subsequent analyses, data reported for the time closest to macroscopic failure reflect the fracture network development that occurs from the second-to-last $\left(t_{\mathrm{f}-2}\right)$ and final $\left(t_{\mathrm{f}-1}\right)$ scan acquired in the experiment, where $t_{\mathrm{f}}$ is the time of macroscopic failure.

Throughout stages I-II in each experiment, both the number and total volume of preexisting and nucleating fractures increase with increasing strain at comparable levels (Fig. 4). We consider the rate of growth to be the increase in the number or volume of fractures per strain increment. An increase or decrease in the rate of growth thus marks an acceleration or deceleration, respectively, in fracture growth in terms of number or volume. During the transition from stage II to III at yielding, the number and volume of the preexisting fractures accelerate, whereas the number and volume of nucleating fractures do not accelerate as quickly. Due to this bifurcation in acceleration, the number and volume of preexisting fractures exceed those of the nucleating fractures at the end of stage III and through stage IV prior to failure (Fig. 4a, b). At the end of stage IV, the volume of preexisting fractures exceeds the volume of newly nucleating fractures by several orders of magnitude (Fig. $4 \mathrm{~b}, \mathrm{c}$ ). In particular, at the end of stage IV the volume of newly nucleating fractures is $1 \%$, $<1 \%$, and $13 \%$ of the volume of preexisting fractures in experiment no. 3 , no. 5 , and no. 4 , respectively. Overall, preexisting fracture propagation dominates fracture nucleation in the monzonite rocks deformed to failure.

Our results show that while the acceleration in the number of preexisting fractures coincides with the yield point, the acceleration in the volume of preexisting fractures becomes significant only during stage IV, when macroscopic failure is imminent. This trend may also occur for nucleating fractures, but the number of nucleating fractures identified near the yield point is too low to draw the conclusion with confidence. Finally, the function of preexisting fracture volume relative to axial strain is approximately constant in log-linear strain-volume space (Fig. 4c), indicating an exponential increase in total volume as a function of axial strain. The exponents of the best-fit exponential functions of the preexisting fracture volume relative to axial strain range from 725-2000 for the three experiments, with $R^{2}$ values between the best-fit functions and the data of $0.85-0.98$.

\subsection{Isolated fracture propagation and fracture coalescence}

To assess the influence of isolated fracture propagation relative to coalescence on fracture network development, we develop a method to recognize when fractures develop from the merger of two or more fractures (i.e., coalesce) or from the lengthening, opening, or closing of only one fracture (i.e., isolated propagation). Figure 5 shows the number and total volume of fractures identified as developing from two or more fractures (i.e., coalescing) or from only one preexisting fracture (i.e., propagating). We use the short-hand term propagating to indicate fractures that grow in isolation, but we note that fractures identified as coalescing also propagate before or while they merge.

The number of propagating fractures is larger than the number of coalescing fractures throughout loading in each experiment (Fig. 5a). The number and volume of propagating fractures accelerate throughout stages II-IV. In contrast, the number and volume of coalescing fractures only appear to accelerate following yielding throughout stages III-IV. Overall, the differences in the number and volume of propagating and coalescing fractures grow larger during stages I-III.

At the end of stage IV, immediately preceding macroscopic failure, the total volume of coalescing fractures exceeds the total volume of propagating fractures in the nominally dry experiments (experiment no. 3 and no. 5). During this stage, the volume of propagating fractures is $44 \%$ or $23 \%$ of the volume of coalescing fractures for experiment no. 3 and no. 5 , respectively. In contrast, in the watersaturated experiment (no. 4), the total volume of coalescing fractures never exceeds the total volume of propagating fractures. Immediately preceding macroscopic failure, the volume of propagating fractures is about 7 times higher than the volume of coalescing fractures in this experiment. Thus, 

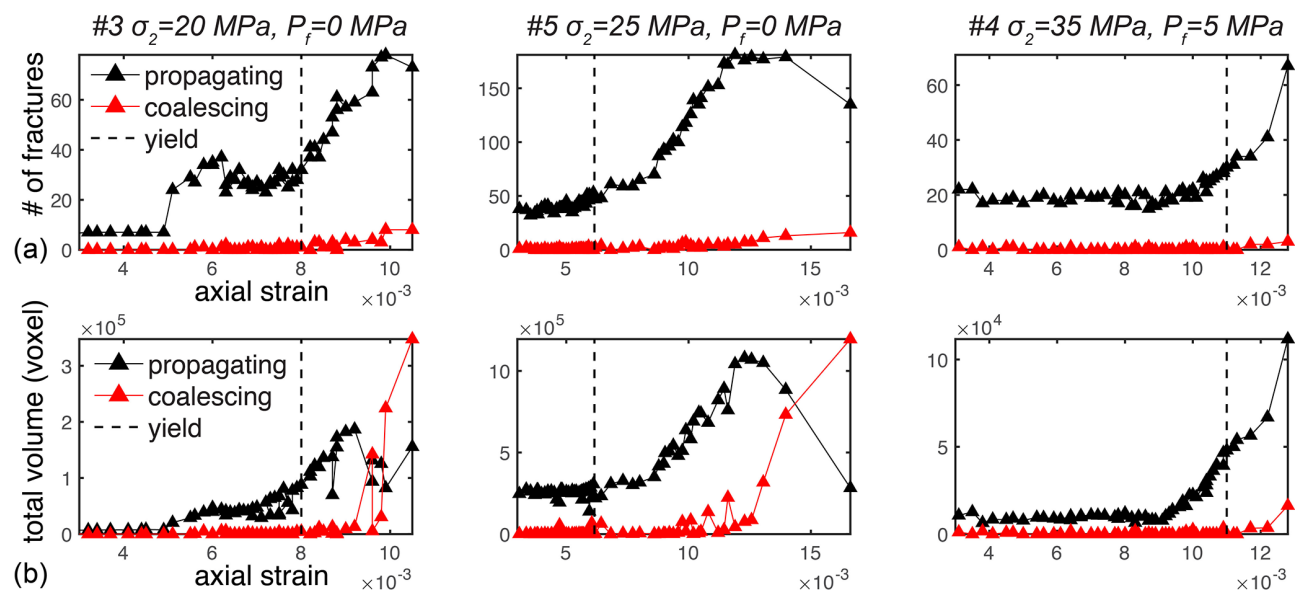

Figure 5. The varying influence of preexisting fracture coalescence and propagation. (a) The number of fractures propagating in isolation (black) and coalescing (red). (b) The total volume of fractures propagating in isolation or coalescing. Prior to macroscopic failure, the total volume of propagating fractures decreases and the total volume of coalescing fractures increases in the nominally dry experiments (no. 3 and no. 5), indicating the dominance of coalescence rather than isolated propagation. In contrast, in the water-saturated experiments, propagating fractures dominate throughout loading.

water-saturated conditions and higher confining stress appear to promote fracture propagation and suppress coalescence.

\subsection{Disperse and localized fracture growth}

To characterize the influence of localization and stress perturbations on fracture network development, we identify the fractures that gain and lose volume from one loading step to the next, i.e., grow or close, and whether they are located near or far from another fracture. Analytical formulations of LEFM with the stress intensity factor suggest that fractures perturb their local stress field to a distance on the order of their length (e.g., Chinnery and Petrak, 1968; Segall and Pollard, 1980; Atkinson, 1987; Scholz et al., 1993; Davy et al., 2010, 2013). A corollary of this concept is that fractures that are within one fracture length of other (perturbing) fractures may be more likely to grow and less likely to close. This behavior will only be true if the local stress perturbation is favorable for growth. In contrast, local stress perturbations can also produce stress fields that hinder fracture growth, i.e., stress shadows. In this case, if a fracture lies in a stress shadow, it should be less likely to grow and perhaps more likely to close. We test these inferences here. In particular, we track the number of growing and closing fractures that do (i.e., near) and do not (i.e., far) have other fractures within one fracture length of them at each stress step (Fig. 6). For example, if one fracture (fracture no. 1) is located within $y$ distance of another fracture (no. 2) with length $y$, then fracture no. 1 is counted in the near category.

The number of growing fractures matches the number of closing fractures in stages I-II early in loading (Fig. 6a). During stage III after yielding, the number of growing fractures accelerates, while the number of closing fractures remains at similar values. The number of growing fractures that are located near other fractures (within a fracture length of them) increases with loading (Fig. 6b). In contrast, the number of growing fractures that are far from other fractures remains roughly constant throughout loading. These varying trends produce two patterns of fracture growth before and after the yield point. In stages I-II before yielding, the number of growing fractures located far from other fractures exceeds or is similar to the number of growing fractures located near other fractures. These observations suggest that fractures located closer to other fractures are not more likely to grow than fractures spread further apart, indicating that stress concentrations produced by developing fractures do not appreciably influence fracture development preceding yielding. In stages III-IV after yielding, however, the number of growing fractures located near other fractures increasingly exceeds the number of growing fractures located far from other fractures. At the end of stage IV immediately preceding macroscopic failure in all three experiments, the number of growing fractures located near others is $3-5$ times higher than the number growing far from others. We note that increasing fracture length will tend to limit the available volume in which fractures classified as far may develop. With this caveat, when macroscopic failure becomes imminent, the stress concentrations produced by growing fractures appear to promote growth rather than suppress it.

The evolution of the number of growing fractures located near others further highlights the influence of coalescence on fracture network development (Fig. 6b). The number of these fractures decreases in the final loading steps just before failure in the dry experiments (no. 3 and no. 5). In contrast, the number of these fractures continually increases in the watersaturated experiment (no. 4). Fracture coalescence reduces 

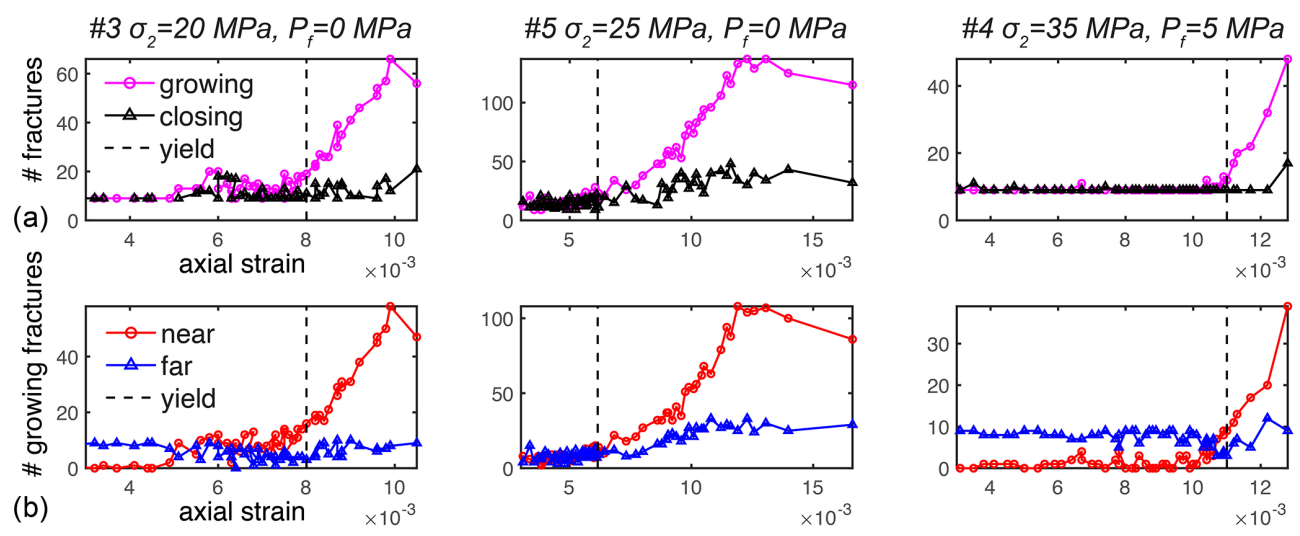

Figure 6. The influence of stress perturbations on fracture growth. (a) The number of growing (magenta circles) and closing (black triangles) fractures. (b) The number of growing fractures that do (near, red circles) and do not (far, blue triangles) have other fractures within one fracture length of them throughout loading. If one fracture (fracture no. 1) is located within $y$ distance of another fracture (no. 2) with length $y$, then fracture no. 1 is counted in the near category. Following the macroscopic yield point, the number of growing fractures located near other fractures exceeds the number located far from others. This observation suggests that the local perturbations of the stress field produced by fracture growth tend to promote the growth of other fractures following yielding.

the total number of fractures as many smaller fractures merge into a few larger fractures.

\section{Discussion}

\subsection{The competition between fracture nucleation and preexisting fracture propagation}

In monzonite rocks undergoing brittle failure, preexisting fracture propagation dominates fracture nucleation after yielding (Fig. 4). Our results indicate that increasing differential stress promotes the dominance of preexisting fracture development rather than nucleation. As the fractures lengthen and open under increasing differential stress, the stress intensity factors at their tips increase (Isida, 1971) and thereby further promote propagation. As deformation localizes among several larger fractures, the energetic cost of propagating preexisting fractures may become less than the cost of nucleating new fractures (e.g., Del Castello and Cooke, 2007; Herbert et al., 2015). Our data support these predictions from the viewpoint of linear elastic fracture mechanics and energy optimization.

In addition to the evolving fracture lengths, mechanical heterogeneities control the location of fracture nucleation and the growth of preexisting fractures. For example, Tapponnier and Brace (1976) documented the fact that fracture development initiates along grain boundaries and healed transgranular fractures in granite, and new transgranular fractures propagate only at higher differential stresses. These mechanical controls influence the ability of fractures to nucleate and propagate following nucleation: fractures can arrest at grain boundaries and mechanical contacts, depending on the degree of stress transfer across such interfaces (e.g., Tapponnier and Brace, 1976; Cooke and Underwood,
2001; McBeck et al., 2019a, b). Granular rocks may contain mechanical heterogeneities that concentrate shear and/or tensile stresses more effectively than monzonite, which consists of an interlocking crystalline structure with more homogeneous mechanical properties. For example, numerical discrete-element models of sandstone indicate that the degree of strength heterogeneity between grain boundaries and intragranular material controls the proportion of fractures that nucleate at grain boundaries and those that nucleate within grains (McBeck et al., 2019b). Thus, in a given sandstone volume there will likely be a greater number of sites of significant stress concentrations than in a monzonite or granite volume and thereby a larger number of sites suitable for fracture nucleation. Consequently, we may expect a greater dominance of nucleation in sandstone and other rocks with strong strength heterogeneity than observed in monzonite rocks.

\subsection{The competition between isolated fracture propagation and coalescence}

Tracking the volume of fractures that coalesce from several fractures and those that propagate in isolation without merging indicates that isolated propagation dominates coalescence throughout most of the deformation process preceding macroscopic failure (Fig. 5). Preceding macroscopic failure, our results suggest that the presence of fluid and the magnitude of confining stress may affect the competition between isolated propagation and coalescence. We deformed the water-saturated sample (experiment no. 4) with the highest effective confining stress; the confining pressure minus the pore fluid pressure was $30 \mathrm{MPa}$. In this experiment, the total volume of coalescing fractures was $<10 \%$ of the volume of propagating fractures immediately preceding macroscopic failure (Fig. 5b). In contrast, in the experiments deformed at 
lower confining stress (20 and $25 \mathrm{MPa}$ in experiment no. 3 and no. 5, respectively) and dry conditions, the total volume of coalescing fractures was at least twice the volume of the propagating fractures preceding failure. This difference in behavior suggests that dry conditions and lower confining stress promote coalescence rather than isolated propagation.

Many observations indicate that the magnitude of confining stress influences fracture development, similar to our observation that the confining stress influences the proportion of propagating and coalescing fractures. In the end-member case when a rock undergoes uniaxial compression (i.e., zero confinement), experiments show that opening mode and tensile failure dominate deformation with little evidence of shear deformation (e.g., Lin et al., 2015). Tapponnier and Brace (1976) observed few shear fractures in triaxial experiments on Westerly granite under $50 \mathrm{MPa}$ of confining stress. With increasing confinement, fractures can appear to rotate from the orientation preferred under uniaxial compression conditions (parallel to the maximum compression direction) toward the range of orientations predicted by the maximum Coulomb shear stress (e.g., Mair et al., 2002; McBeck et al., 2019a). Analyses often interpret such rotation to indicate an increasing dominance of shear deformation at the expense of tensile deformation. However, such apparent rotation may occur as many individual mode-I fractures link together so that the macroscopic trend of the fault is inclined relative to the maximum compression direction (e.g., Peng and Johnson, 1972; Lockner et al., 1991; Renard et al., 2019a). Consequently, the fracture geometry alone may not indicate the relative proportion of shear and tensile deformation.

Analysis of the moment tensors of acoustic emissions provides further insights into the relative proportion of shear and tensile deformation under varying confining stresses. Analysis of acoustic emissions during triaxial compression suggests that decreasing confining stress promotes tensile failure and opening at the expense of shear failure (e.g., Stanchits et al., 2006). This opening may enable greater access to preexisting fractures than shear deformation, thereby promoting the likelihood of coalescence. For example, mixed-mode fractures may tend to have larger apertures than fractures dominated by shear deformation. Mixed-mode fractures may have larger apertures because opening can increase fracture aperture. As larger apertures can yield greater fracture surface area, mixed-mode failure may result in thicker fractures that provide greater surface area to which other fractures can link than thinner fractures produced predominately by shear. Confining stress tends to reduce the proportion of tensile deformation relative to shear deformation and may thus localize deformation into thinner zones in the absence of cataclastic flow and ductile deformation.

The applied confining pressure in experiment no. 5 was $5 \mathrm{MPa}$ higher than that of experiment no. 3, but these two dry samples show similar proportions of fracture propagation and coalescence. Consequently, it is unlikely that the $5 \mathrm{MPa}$ higher effective stress of experiment no. 4 compared to experiment no. 5 is the primary trigger of the different behaviors observed in these experiments. We suggest that the presence of water is responsible for the transition from isolated propagation to coalescence-dominated fracture network development. We acknowledge that this conclusion rests on only three experiments and further work is required for more robust support of this idea. However, previous work focused on the influence of water on fracture network growth supports this idea. In particular, this work shows that chemical reactions at fracture tips can influence fracture propagation. Such stress corrosion cracking occurs when chemical reactions reduce the fracture toughness and thereby promote crack propagation (e.g., Anderson and Grew, 1977). When water is present, hydrogen bond formation weakens the $\mathrm{Si}-$ $\mathrm{O}$ bond in quartz-rich sandstones, producing water weakening (e.g., Baud et al., 2000). Stress corrosion cracking may thus promote nucleation at the expense of coalescence in the water-saturated monzonite experiment.

Changes in pore fluid pressure can also affect the fracture propagation rate (Ougier-Simonin and Zhu, 2013, 2015). Recent studies show that at the same effective pressure and loading, fault propagation in intact serpentinite is slower in samples with higher pore fluid pressures (French and Zhu, 2017). When a fluid-saturated rock dilates, the pore pressure may drop and thereby reduce the local effective confinement and strengthen the rock, i.e., dilatant hardening (e.g., Brace and Bombolakis, 1963; Rice, 1975; Rudnicki and Chen, 1988; Ikari et al., 2009; Xing et al., 2019; Brantut, 2020). This strengthening can then slow the rate of fracture propagation from dynamic to quasi-stable (Martin III, 1980; French and Zhu, 2017).

Dilatant hardening may influence fracture development in the water-saturated experiment (no. 4) if the evolving permeability of the network is high enough to allow fluid flow at the timescale of the experiment. Using the porosity identified in the tomograms acquired immediately preceding failure, the porosity of the rocks in each experiment ranges from $0.06 \%$ (no. 4) to $0.2 \%$ (no. 3) and $1.6 \%$ (no. 5) at this stage. Following the relationships between porosity and permeability calculated for dynamically fractured monzonite cores (Aben et al., 2020), rocks with $0.06 \%-1.6 \%$ porosity may have permeability of $10^{-16}$ to $10^{-18} \mathrm{~m}^{-2}$. With this range of permeability and dimensions of the rock core, water requires less than a minute to $45 \mathrm{~min}$ to traverse the core from top to bottom (Text S1 of Supplement). Thus, the time interval of the loading steps ( $3 \mathrm{~min}$ ) may allow water to flow between fractures, enabling the effects of stress corrosion cracking and dilatant hardening to operate at least in the final stages preceding failure. Earlier in the experiment, when the porosity and permeability are lower, the lower flow rate may suppress such effects. Further experimental investigations are needed to distinguish between the relative importance of stress corrosion cracking and dilatant hardening for fracture development within water-saturated rocks. 

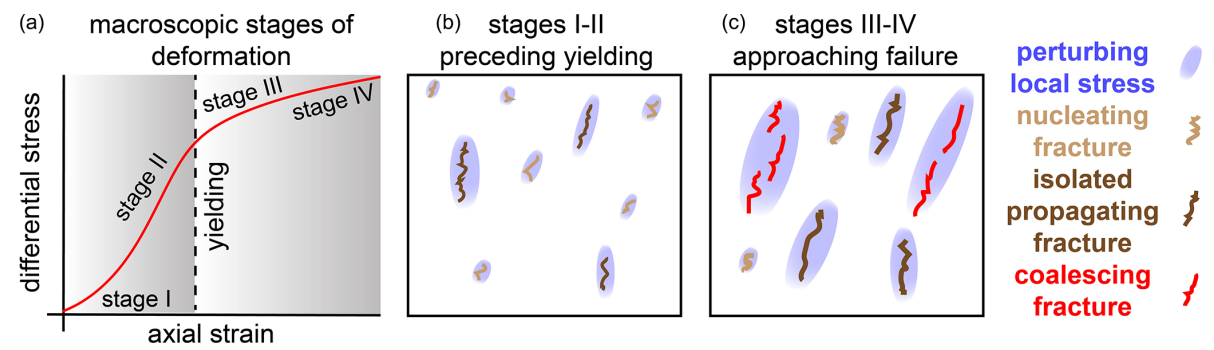

Figure 7. Schematic of varying modes of fracture development observed preceding yielding and approaching macroscopic failure. Nucleating, propagating, and coalescing fractures are shown in light brown, dark brown, and red, respectively. Blue ellipsoids show the approximate extent of the perturbation of the local stress field produced by fracture network development. When the rock experiences lower differential stress and the fracture network is more distributed, (1) similar numbers of new fractures nucleate and preexisting fractures grow, (2) isolated propagation dominates coalescence, and (3) local stress perturbations do not appear to promote fracture growth. When the system approaches macroscopic failure, (1) preexisting fracture propagation dominates new fracture nucleation, (2) coalescence dominates isolated propagation in the experiments with the lowest confining stress and dry conditions, and (3) local stress perturbations promote fracture growth.

These observations and our analyses suggest that the presence of water (producing stress corrosion) and high pore fluid pressure (producing dilatant hardening) promote slower, more isolated fracture network growth rather than faster, coalescence-dominated growth. Understanding the mechanical and chemical conditions that favor one mode of fracture growth over another (e.g., fracture coalescence versus isolated fracture propagation) has important implications in many energy and environmental engineering practices. For example, when their connected porosities are comparable, fracture networks produced by the propagation and coalescence of many small fractures may have lower connectivity, higher tortuosity, and lower permeability than networks consisting of a few large fractures. However, fracture networks consisting of numerous small fractures may be more efficient in shale gas exploration and $\mathrm{CO}_{2}$ sequestration (e.g., Xing et al., 2018).

\subsection{The influence of local stress perturbations on fracture growth}

Clear factors in fracture network development are fracture network density, clustering, and localization. For example, earthquakes are more likely to arrest at the ends of faults that are $>5 \mathrm{~km}$ from another fault (Wesnousky, 2006). Indeed, the distance between fractures is one of the key parameters that predicts the timing of catastrophic failure and whether fractures grow or close from one stress step to the next in Xray tomography triaxial compression experiments on marble, monzonite, and granite rocks (McBeck et al., 2019a, 2020a). Analytical solutions from LEFM provide a mechanical interpretation of these observations. These solutions indicate that a fracture will perturb the local stress field to a distance on the order of their length (e.g., Scholz et al., 1993). Following this idea, we may use the number of growing fractures located within this threshold to determine if stress perturbations produced by growing fractures tend to promote or hinder growth.
Our observations suggest that local stress perturbations produced by growing fractures promote the growth of neighboring fractures during stages III-IV preceding macroscopic failure (Fig. 6), consistent with previous work (e.g., Scholz et al., 1993; Wesnousky et al., 2006; McBeck et al., 2019a, 2020b). During these stages, the number of fractures that grow and are located within one fracture length exceeds the number of fractures that grow and are located outside this threshold. Preceding yielding, however, similar numbers of growing fractures are located both within and outside this threshold. When the fracture network is more diffuse under lower differential stress, the distance between fractures does not appear to influence whether a fracture grows or closes (i.e., Fig. 7). When the fracture network becomes more clustered, the distance between fractures appears to influence whether a fracture grows or closes. Our results highlight the conditions under which stress perturbations influence growth in rocks under triaxial compression that host fracture networks with a variety of spatial distributions.

\section{Conclusions}

In situ dynamic X-ray tomography during the triaxial compression of crystalline rocks reveals the competing influence of three modes of fracture network development: (1) nucleation, (2) isolated propagation, and (3) coalescence. We find that the influence of these modes evolves throughout loading, with clear transitions near yielding and macroscopic failure. Preexisting fracture propagation, including isolated propagation and coalescence, becomes the dominant mode of deformation following yielding. Coalescence then becomes the dominant mechanism of fracture network development in dry samples under lower confinements only immediately preceding macroscopic failure. Isolated propagation remains the dominant mechanism throughout loading in a watersaturated sample under higher confinement. Compared to the prediction that fractures promote growth by perturbing their 
local stress field to a distance on the order of their length (e.g., Scholz et al., 1993), our observations only match these expectations in the stages of the experiments between yielding and macroscopic failure. Preceding yielding, however, the fractures that are growing are not significantly closer to other fractures, indicating that their stress perturbations do not promote the growth of neighboring fractures. When the rock experiences lower differential stress and the fracture network is more distributed, (1) similar numbers of new fractures nucleate and preexisting fractures grow, (2) isolated propagation dominates coalescence, and (3) local stress perturbations do not appear to promote fracture growth (Fig. 7). When the rock experiences higher differential stress following yielding, (1) preexisting fracture propagation dominates new fracture nucleation, (2) coalescing fracture volume exceeds the propagating fracture volume in dry samples when macroscopic failure is imminent, and (3) local stress perturbations promote fracture growth.

Data availability. The data are available on the NorStore repository (Renard, 2017, 2018).

Supplement. The supplement related to this article is available online at: https://doi.org/10.5194/se-12-375-2021-supplement.

Author contributions. JAM and FR performed the experiments, analyzed results, and wrote the paper. WZ analyzed results and wrote the paper.

Competing interests. The authors declare that they have no conflict of interest.

Special issue statement. This article is part of the special issue "Thermo-hydro-mechanical-chemical (THMC) processes in natural and induced seismicity". It is a result of the The 7th International Conference on Coupled THMC Processes, Utrecht, Netherlands, 35 July 2019.

Acknowledgements. We thank Elodie Boller, Paul Tafforeau, and Alexander Rack for providing advice on the design of the tomography setup, Benoît Cordonnier for experimental expertise, and Sanchez Technology for building the deformation apparatus. The Research Council of Norway (awards 272217 to François Renard and 300435 to Jessica A. McBeck) and the US National Science Foundation (EAR-1761912 to Wenlu Zhu) funded this work. The European Synchrotron Radiation Facility allocated beam time (Long Term Proposal ES-295). We thank guest editor André Niemeijer, reviewer Frans Aben, and two anonymous reviewers for suggestions that improved this paper.
Financial support. This research has been supported by the Research Council of Norway (grant nos. 272217, 300435) and the US National Science Foundation (grant no. EAR-1761912).

Review statement. This paper was edited by Andre R. Niemeijer and reviewed by Franciscus Aben and two anonymous referees.

\section{References}

Aben, F. M., Doan, M.-L., and Mitchell, T. M.: Variation of hydraulic properties due to dynamic fracture damage: Implications for fault zones, J. Geophys. Res.-Sol. Ea., 125, e2019JB018919, https://doi.org/10.1029/2019JB018919, 2020.

Aben, F. M., Doan, M. L., Mitchell, T. M., Toussaint, R., Reuschlé, T., Fondriest, M., Gratier J-P., and Renard, F.: Dynamic fracturing by successive coseismic loadings leads to pulverization in active fault zones. J. Geophys. Res.-Sol. Ea., 121, 2338-2360, 2016.

Anderson, O. L. and Grew, P. C.: Stress corrosion theory of crack propagation with applications to geophysics, Rev. Geophys. Space Phys., 15, 77-104, 1977.

Atkinson, B. K.: Subcritical crack growth in geological materials, J. Geophys. Res.-Sol. Ea., 89, 4077-4114, 1984.

Atkinson, B. K.: Fracture Mechanics of Rock, 534, Academic, London, 1987.

Baud, P., Zhu, W., Wong, T.-F.: Failure mode and weakening effect of water on sandstone, J. Geophys. Res., 105, 16371-16389, 2000.

Blanpied, M. L., Marone, C. J., Lockner, D. A., Byerlee, J. D., and King, D. P.: Quantitative measure of the variation in fault rheology due to fluid-rock interactions, J. Geophys. Res.-Sol. Ea., 103, 9691-9712, 1998.

Brace, W. F. and Bombolakis, E. G.: A note on brittle crack growth in compression, J. Geophys. Res., 68, 3709-3713, https://doi.org/10.1029/JZ068i012p03709, 1963.

Brantut, N.: Dilatancy-induced fluid pressure drop during dynamic rupture: Direct experimental evidence and consequences for earthquake dynamics, Earth Planet. Sci. Lett., 538, 116179, https://doi.org/10.1016/j.epsl.2020.116179, 2020.

Cartwright-Taylor, A., Main, I. G., Butler, I. B., Fusseis, F., Flynn, M., and King, A.: Catastrophic Failure: How and When? Insights From 4-D In Situ X-ray Microtomography, J. Geophys. Res.-Sol. Ea., 125, e2020JB019642, https://doi.org/10.1029/2020JB019642, 2020.

Chinnery, M. A. and Petrak, J. A.: The dislocation fault model with a variable discontinuity, Tectonophysics, 5, 513-529, https://doi.org/10.1016/0040-1951(68)90008-5, 1968.

Cooke, M. L. and Underwood, C. A.: Fracture termination and stepover at bedding interfaces due to frictional slip and interface opening, J. Struct. Geol., 23, 223-238, 2001.

Cui, G., Wang, Y., Rui, Z., Chen, B., Ren, S., and Zhang, L.: Assessing the combined influence of fluid-rock interactions on reservoir properties and injectivity during $\mathrm{CO}_{2}$ storage in saline aquifers, Energy, 155, 281-296, 2018.

Crider, J. G. and Peacock, D. C.: Initiation of brittle faults in the upper crust: a review of field observations, J. Struct. Geol., 26, 691-707, 2004. 
Davy, P., Goc, R., Darcel, C., Bour, O., Dreuzy, J., and Munier, R.: A likely universal model of fracture scaling and its consequence for crustal hydromechanics, J. Geophys. Res.-Sol. Ea., 115, 113, https://doi.org/10.1029/2009jb007043, 2010.

Davy, P., Le Goc, R., and Darcel, C.: A model of fracture nucleation, growth and arrest, and consequences for fracture density and scaling, J. Geophys. Res.-Sol. Ea., 118, 1393-1407, https://doi.org/10.1002/jgrb.50120, 2013.

Del Castello, M. and Cooke, M. L.: Underthrusting-accretion cycle: Work budget as revealed by the boundary element method, J. Geophys. Res.-Sol. Ea., 112, B12404, https://doi.org/10.1029/2007JB004997, 2007.

Du, Y. and Aydin, A.: The maximum distortional strain energy density criterion for shear fracture propagation with applications to the growth paths of en echelon faults, Geophys. Res. Lett., 20, 1091-1094, 1993.

Fattaruso, L. A., Cooke, M. L., Dorsey, R. J., and Housen, B. A.: Response of deformation patterns to reorganization of the southern San Andreas fault system since ca. 1.5 Ma, Tectonophysics, 693, 474-488, 2016.

French, M. E. and Zhu, W.: Slow fault propagation in serpentinite under conditions of high pore fluid pressure, Earth Planet. Sci. Lett., 473, 131-140, 2017.

Frery, E., Gratier, J. P., Ellouz-Zimmerman, N., Loiselet, C., Braun, J., Deschamps, P., Blamart, D., Hamelin, B., and Swennen, R., Evolution of fault permeability during episodic fluid circulation: Evidence for the effects of fluid-rock interactions from travertine studies (Utah-USA), Tectonophysics, 651, 121-137, 2015.

Griffith, A. A.: VI. The phenomena of rupture and flow in solids, Philos. T. R. Soc. S. A, containing papers of a mathematical or physical character, 221, 163-198, 1921.

Herbert, J. W., Cooke, M. L., Souloumiac, P., Madden, E. H., Mary, B. C., and Maillot, B.: The work of fault growth in laboratory sandbox experiments, Earth Planet. Sci. Lett., 432, 95-102, 2015.

Hickman, S., Sibson, R., and Bruhn, R.: Introduction to special section: Mechanical involvement of fluids in faulting, J. Geophys. Res.-Sol. Ea., 100, 12831-12840, 1995.

Ikari, M. J., Saffer, D. M., and Marone, C.: Frictional and hydrologic properties of clay-rich fault gouge, J. Geophys. Res.-Sol. Ea., 114, B05409, https://doi.org/10.1029/2008JB006089, 2009.

Irwin, G.: Analysis of stresses and strains near the end of a crack traversing a plate, J. Appl. Mech., 24, 361-364, 1957.

Isida, M.: Effect of width and length on stress intensity factors of internally cracked plates under various boundary conditions, Int. J. Fract. Mech., 7, 301-316, 1971.

Jackson, C. A. L. and Rotevatn, A.: 3D seismic analysis of the structure and evolution of a salt-influenced normal fault zone: a test of competing fault growth models, Journal of Structural Geology, 54, 215-234, 2013.

Jaeger, J. C., Cook, N. G. W., and Zimmerman, R.: Rock mechanics, Fundamentals of Rock Mechanics, Chapman and Hall, London, 1979.

Kandula, N., Cordonnier, B., Boller, E., Weiss, J., Dysthe, D. K., and Renard, F.: Dynamics of microscale precursors establish brittle-compressive failure as a critical phenomenon in Carrara marble, J. Geophys. Res., 124, 6121-6139, https://doi.org/10.1029/2019JB017381, 2019.
Kawakata, H., Cho, A., Yanagidani, T., and Shimada, M.: The observations of faulting in Westerly granite under triaxial compression by X-ray CT scan, Int. J. Rock Mech. Min. Sci., 34, 151-e1151-e12, 1997.

Lamy-Chappuis, B., Angus, D., Fisher, Q., Grattoni, C., and Yardley, B. W.: Rapid porosity and permeability changes of calcareous sandstone due to $\mathrm{CO}_{2}$-enriched brine injection, Geophys. Res. Lett., 41, 399-406, 2014.

Lin, P., Wong, R. H., and Tang, C. A.: Experimental study of coalescence mechanisms and failure under uniaxial compression of granite containing multiple holes, Int. J. Rock Mech. Min. Sci., 77, 313-327, 2015.

Lockner, D., Byerlee, J. D., Kuksenko, V., Ponomarev, A., and Sidorin, A.: Quasi-static fault growth and shear fracture energy in granite, Nature, 350, 39-42, 1991.

Madden, E. H., Cooke, M. L., and McBeck, J.: Energy budget and propagation of faults via shearing and opening using work optimization, J. Geophys. Res.-Sol. Ea., 122, 6757-6772, 2017.

Mair, K., Elphick, S., and Main, I.: Influence of confining pressure on the mechanical and structural evolution of laboratory deformation bands, Geophys. Res. Lett., 29, 49-51, 2002.

Mansfield, C. and Cartwright, J.: Fault growth by linkage: observations and implications from analogue models. J. Struct. Geol., 23, 745-763, 2001.

Martin III, R. J.: Pore pressure stabilization of failure in Westerly granite, Geophys. Res. Lett., 7, 404-406, 1980.

Martin, C. D. and Chandler, N. A.: The progressive fracture of Lac du Bonnet granite, Int. J. Rocj. Mech. Min., 31, 643-659, 1994.

Mary, B. C. L., Maillot, B., and Leroy, Y. M.: Deterministic chaos in frictional wedges revealed by convergence analysis, Int. J. Num. Anal. Met., 37, 3036-3051, 2013.

McBeck, J. A., Aiken, J. M., Mathiesen, J., Ben-Zion, Y., and Renard, F., Deformation precursors to catastrophic failure in rocks, Geophys. Res. Lett., e2020GL090255, https://doi.org/10.1029/2020GL090255, 2020a.

McBeck, J., Cooke, M., and Fattaruso, L.: Predicting the propagation and interaction of frontal accretionary thrust faults with work optimization, Tectonophysics, 228461, https://doi.org/10.1016/j.tecto.2020.228461, 2020 b.

McBeck, J., Cooke, M., and Madden, E.: Work optimization predicts the evolution of extensional step overs within anisotropic host rock: Implications for the San Pablo Bay, CA, Tectonics, 36, 2630-2646, 2017.

McBeck, J., Kandula, N., Aiken, J. M., Cordonnier, B., and Renard, F.: Isolating the Factors That Govern Fracture Development in Rocks Throughout Dynamic In Situ X-Ray Tomography Experiments, Geophys. Res. Lett., 46, 11127-11135, 2019 a.

McBeck, J., Mair, K., and Renard, F.: How porosity controls macroscopic failure via propagating fractures and percolating force chains in porous granular rocks, J. Geophys. Res.-Sol. Ea., 124, 9920-9939, 2019b.

Müller, G.: Starch columns: Analog model for basalt columns, J. Geophys. Res.-Sol. Ea., 103, 15239-15253, 1998.

Nemat-Nasser, S. and Horii, H.: Compression-induced nonplanar crack extension with application to splitting, exfoliation, and rockburst, J. Geophys. Res.-Sol. Ea., 87, 6805-6821, 1982.

Okubo, C. H., and Schultz, R. A.: Evolution of damage zone geometry and intensity in porous sandstone: insight gained from strain energy density, J. Geol. Soc. London, 162, 939-949, 2005. 
Olson, J. E.: Joint pattern development: Effects of subcritical crack growth and mechanical crack interactionm J. Geophys. Res.-Sol. Ea., 98, 12251-12265, 1993.

Olson, E. L. and Cooke, M. L.: Application of three fault growth criteria to the Puente Hills thrust system, Los Angeles, California, USA, J. Struct. Geol., 27, 1765-1777, 2005.

Ougier-Simonin, A. and Zhu, W.: Effect of pore pressure build-up on slowness of rupture propagation, J. Geophys. Res., 120, 79667985, https://doi.org/10.1002/2015JB012047, 2015.

Ougier-Simonin, A. and Zhu, W.: Effects of pore fluid pressure on slip behaviors: an experimental study, Geophys. Res. Lett., 40, 2619-2624, https://doi.org/10.1002/grl.50543, 2013.

Paterson, M. S. and Wong, T. F.: Experimental rock deformation-the brittle field, Springer Science and Business Media, New York, 2005.

Peng, S. and Johnson, A. M.: Crack growth and faulting in cylindrical specimens of Chelmsford granite, Int. J. Rock Mech. Min. Sci., 9, 37-86, 1972.

Pollard, D. D. and Aydin, A.: Progress in understanding jointing over the past century, Geol. Soc. Am. Bull., 100, 1181-1204, 1988.

Reches, Z. E., and Lockner, D. A.: Nucleation and growth of faults in brittle rocks, J. Geophys. Res.-Sol. Ea., 99, 18159-18173, 1994.

Renard, F.: Critical evolution of damage towards system size failure in a crystalline rock [Data set], Norstore, https://doi.org/10.11582/2017.00025, 2017.

Renard, F.: Volumetric and shear processes in crystalline rock during the approach to faulting [Data set], Norstore, https://doi.org/10.11582/2018.00023, 2018.

Renard, F., Cordonnier, B., Dysthe, D. K., Boller, E., Tafforeau, P., and Rack, A.: A deformation rig for synchrotron microtomography studies of geomaterials under conditions down to $10 \mathrm{~km}$ depth in the Earth, J. Synchrotron Radiat., 23, 1030-1034, 2016.

Renard, F., Gratier, J. P., and Jamtveit, B.: Kinetics of crack-sealing, intergranular pressure solution, and compaction around active faults, J. Struct. Geol., 22, 1395-1407, 2000.

Renard, F., McBeck, J., Cordonnier, B., Zheng, X., Kandula, N., Sanchez, J. R., Kobchenko, M., Noiriel, C., Zhu, W., Meaken, P., and Fusseis, F.: Dynamic in situ three-dimensional imaging and digital volume correlation analysis to quantify strain localization and fracture coalescence in sandstone, Pure Appl. Geophys., 176, 1083-1115, 2019a.

Renard, F., McBeck, J., Kandula, N., Cordonnier, B., Meakin, P., and Ben-Zion, Y.: Volumetric and shear processes in crystalline rock approaching faulting, P. Natl. Acad. Sci. USA, 116, 1623416239, https://doi.org/10.1073/pnas.1902994116, 2019b.
Renard, F., Weiss, J., Mathiesen, J., Ben-Zion, Y., Kandula, N., and Cordonnier, B.: Critical evolution of damage toward system-size failure in crystalline rock, J. Geophys. Res.-Sol. Ea., 123, 19691986, 2018.

Rice, J. R.: On the stability of dilatant hardening for saturated rock masses, J. Geophys. Res., 80, 1531-1536. https://doi.org/10.1029/JB080i011p01531, 1975.

Rudnicki, J. W. and Chen, C. H.: Stabilization of rapid frictional slip on a weakening fault by dilatant hardening, J. Geophys. Res., 93, 4745-4757, https://doi.org/10.1029/JB093iB05p04745, 1988.

Saeedi, A., Delle Piane, C., Esteban, L., and Xie, Q.: Flood characteristic and fluid rock interactions of a supercritical $\mathrm{CO}_{2}$, brine, rock system: South West Hub, Western Australia, Int. J. Green. Gas Cont., 54, 309-321, 2016.

Sausse, J., Jacquot, E., Fritz, B., Leroy, J., and Lespinasse, M.: Evolution of crack permeability during fluid-rock interaction, Example of the Brezouard granite (Vosges, France), Tectonophysics, 336, 199-214, 2001.

Scholz, C. H., Dawers, N. H., Yu, J. Z., Anders, M. H., and Cowie, P. A.: Fault growth and fault scaling laws: preliminary results. J. Geophys. Res.-Sol. Ea., 98, 21951-21961, 1993.

Segall, P. and Pollard, D. D.: Mechanics of discontinuous faults, J. Geophys. Res., 85, 4337-4350, https://doi.org/10.1029/JB085iB08p04337, 1980.

Stanchits, S. Vinciguerra, S., and Dresen, G.: Ultrasonic velocities, acoustic emission characteristics and crack damage of basalt and granite, Pure Appl. Geophys., 163, 975-994, 2006.

Tapponnier, P. and Brace, W. F.: Development of stress-induced microcracks in Westerly granite, Int. J. Rock Mech. Min., 13, 103112, 1976.

Tenthorey, E., Cox, S. F., and Todd, H. F.: Evolution of strength recovery and permeability during fluid-rock reaction in experimental fault zones, Earth Planet. Sci. Lett., 206, 161-172, 2003.

Wesnousky, S. G.: Predicting the endpoints of earthquake ruptures, Nature, 444, 358-360, 2006.

Xing, T., Zhu, W., French, M., and Belzer, B.: Stabilizing Effect of High Pore Fluid Pressure on Slip Behaviors of Gouge-bearing Faults, J. Geophys. Res.-Sol. Ea., 124, 9526-9545, 2019.

Xing, T., Zhu, W., Fusseis, F., and Lisabeth, H.: Generating porosity during olivine carbonation via dissolution channels and expansion cracks, Solid Earth, 9, 879-896, https://doi.org/10.5194/se9-879-2018, 2018.

Zheng, X., Cordonnier, B., McBeck, J., Boller, E., Jamtveit, B., Zhu, W., and Renard, F.: Mixed-mode strain localization generated by hydration reaction at crustal conditions, J. Geophys. Res.-Sol. Ea., 124, 2019. 